\title{
Importance Analysis of Influencing Factors on Cloud Service Composition Flexibility from Service Provider Angle
}

\author{
Kai Liu", a , Xiaotao Yang ${ }^{2, \text { b }}$ \\ 1,2 Jiangsu University of Science and Technology, Zhenjiang 212003, China \\ ascbwcg@163.com, ${ }^{\mathrm{b}}$ cherishyxt@163.com
}

Key words: Service provider; the Influencing factors; Flexibility; Bayesian networks; Importance analysis

\begin{abstract}
The influencing factors of cloud service composition flexibility were analyzed from service provider angle; A Bayesian network model was built to analyze the importance of influencing factors on cloud service composition flexibility from service provider angle, and the critical and non-critical factors for the service provider were identified, which put the groundwork for the subsequently monitoring of the exceptional factors and the management of the flexibility of cloud service composition. Finally, use a shipbuilding enterprise which is constructing service platform for Cloud Manufacture as an example to conduct an application analysis.
\end{abstract}

\section{Introduction}

Cloud Manufacturing (CM) is a new model of manufacturing network which is highly-efficient and service-oriented $^{[1]}$. In this model, service providers can put their unused manufacturing resource to the cloud pool in the form of cloud service. The operator of could manufacturing platform(CMP) then do centralized optimization management to them, and manufacturing task demanders can get their manufacturing service from the cloud pool directly ${ }^{[2-3]}$. The emergence of CM gives us a new way to achieve synergies of information and add the value of manufacturing resource.

Because the demands of manufacturing task demanders are becoming diversified, we need cloud service composition (CSC) to meet the task demands. In the whole life cycle of cloud service composition, there are many uncertain factors influencing the dynamic composition and execution of CSC, which will result in the failure of meeting the needs. In order to ensure the smooth and successful composition of cloud service, CSC should possess the ability of adjusting the composition schemes quickly and executing user demands successfully when uncertain influencing factors occur. It means CSC should have flexibility. This paper focuses on the analysis of influencing factors on cloud service composition flexibility (CSCF) and the importance analysis of these factors. Standing in the perspective of service provider, this paper will analyze and classify influencing factors on service composition flexibility. A Bayesian network model was built to do importance analysis of related influencing factors, and the critical and non-critical influencing factors on CSCF from service provider angle will be identified. This will put the groundwork for the subsequently monitoring of the exceptional factors and the management of the flexibility of cloud service composition. 


\section{Influencing Factors of CSCF from Service Provider Angle}

$\mathrm{Tao}^{[4]}$ pointed out that cloud service composition flexibility (CSCF) is the ability to response the external or internal dynamic changes in the life-cycle of cloud service composition (CSC), and the flow of CSC can be autonomously dynamic reconfigured to complete the tasks. The influencing factors on CSCF refer to all the related factors which will influence the capability of CSC to adjust composition schemes quickly and to execute the task demands successfully. Tao defined the life circle of cloud service composition with three phases, before implementation, in implementation, after implementation. They divided related influencing factors on CSCF into five categories: the change of resource service, the change of user demand, the change of quality of service, the change of application process and others. In fact, the way to categorize influencing factors on CSCF is comparatively unilateral. It did not do niche-targeting and detailed analysis on influencing factors on CSCF from the perspective of three basic role participants of CM. Zhu and other scholars ${ }^{[5-8]}$ mentioned the conception of flexibility and influencing factors of flexibility, but they did not analyze influencing factors on CSCF in detail.

In the whole process of cloud manufacturing application service, there are three basic participant roles: service provider, operator of CMP and task demander. In the dynamic opening environment of $\mathrm{CM}$, there are many abnormal conditions which can't be expected. In practical application, the reasons why three basic participant roles monitor influencing factors are not exactly the same, and the factors they pay special attention to are not exactly the same from their own angle. According to literature reading, investigation, expert experience, combined with the uncertainty and instability and other features of manufacturing cloud service composition, this paper deeply analyzed the influencing factors on CSCF from service provider angle. This paper divided the influencing factors on service composition flexibility which service providers pay special attention to in practical application into six categories: change of own resources(COR), change of other providers' resources(COPR), breakdown of could manufacturing platform(BCMP), post service evaluation from users(PSEU), change of task demanders' requirement(CTDR) and others. These factors are all influencing factors on CSCF from service provider angle, and to some extent they all prevent cloud manufacturing service platform from executing cloud service composition and accomplishing tasks with high efficiency and high quality. Fig.1 demonstrates influencing factors on cloud service composition flexibility from service provider angle.

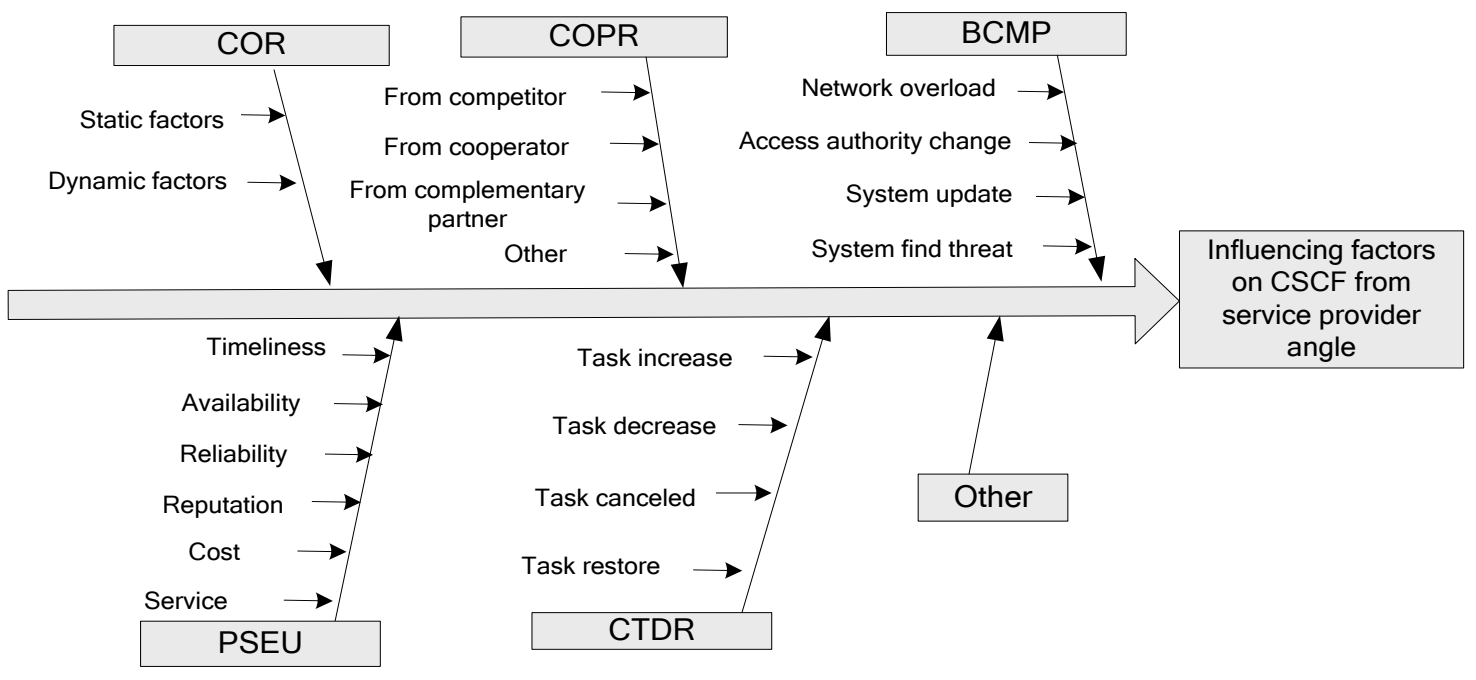

Figure 1.Influencing Factors from Service Provider Angle 
There are many influencing factors on cloud service composition flexibility from service provider angle. In order to make it convenient for service provider to monitor flexible factors with target and high efficiency, it is necessary to analyze the importance of related flexible influencing factors, and proceed to identify the critical and non-critical factors form service provider. This would lay the foundation for the disposal of influencing factors on service composition flexibility and the management and elevation of flexibility.

\section{Bayesian Network Model Constructing}

Bayesian Network $^{[9-10]}(\mathrm{BN})$ is a mathematical model, which possesses the reasoning capability of uncertain information. The nodes represent variables and the directed line segments represent causal relationships between variables, and it uses conditional probability table to analyze quantitatively. Data from different sources are mix together in the form of priori information in order to decrease data requirements in the process of statistical analysis.

\section{Bayesian Network Topology of Influencing Factors}

When build a Bayesian network model, the paper assumes the influencing factors have mutual independence and do not influence each other. Based on the mentioned influencing factors on CSCF from service provider angle, a Bayesian network topology is constructed. Structure is as shown in Fig.2:

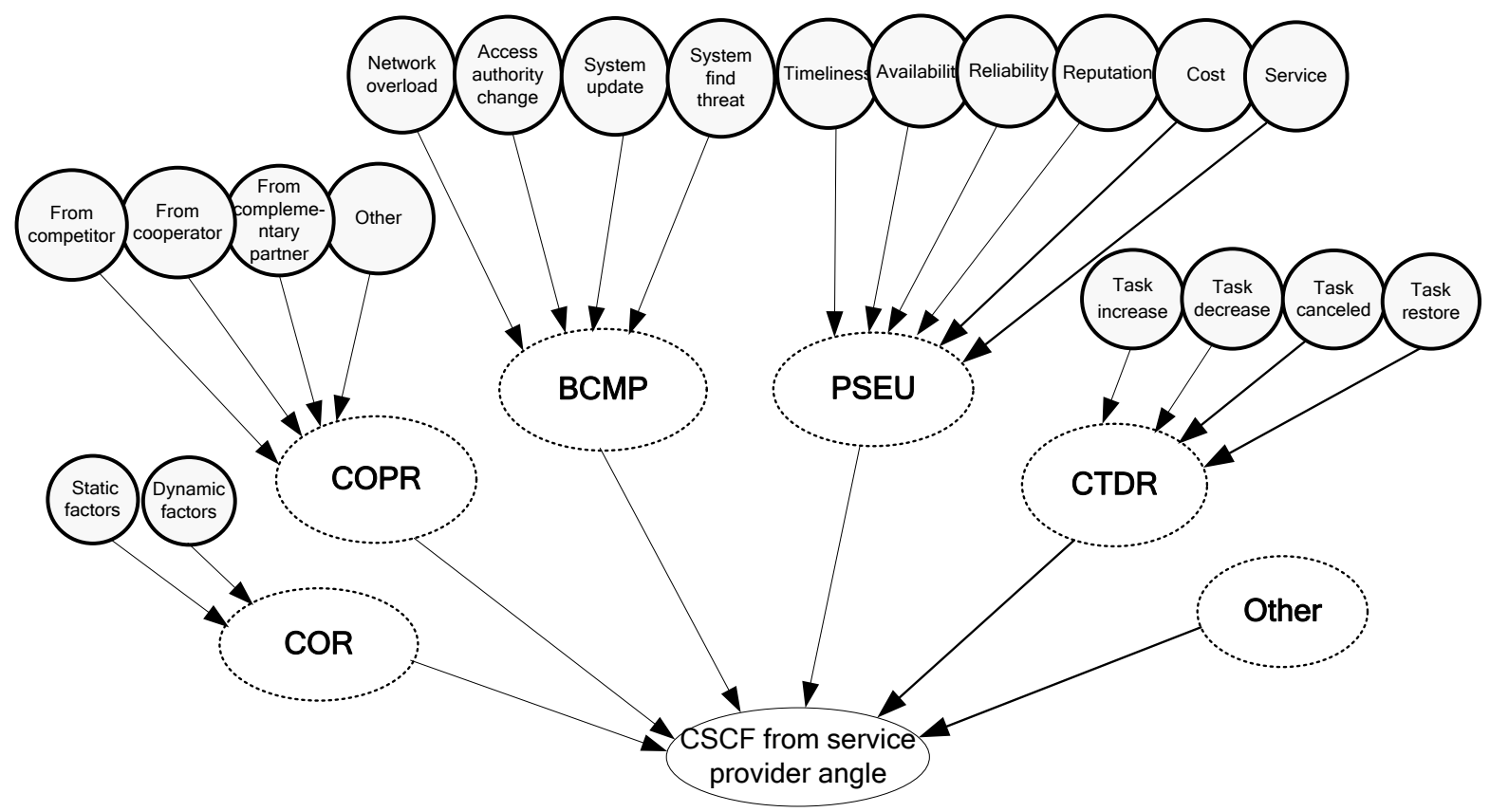

Figure 2.Bayesian Network Topology

\section{Importance Analysis of Influencing Factors}

When influencing factors occur, corresponding nodes need a short process of adjustment. Therefore, when the corresponding node is affected, we can assume that it is in a transient failure state. We set the condition of nodes with two statuses: safety (S) and failure (F). According to the statistical dates, we can know the probability value of each node; then we can calculate the probability of their parent nodes. The probability value expressed as percentage, the formula as follows: 


$$
W_{j T}=\prod_{i=1}^{n} \sigma_{i T}
$$

$$
W_{j F}=1-W_{j T}
$$

In the formula, $\sigma_{i T}$ represents the status of node $i$ safety, $W_{j T}$ represents the status of parent node $j$ safety, $W_{j F}$ represents the status of parent node $j$ failure.

Bayesian network can do importance degree analysis of influencing factors on CSCF. The importance of influencing factors can be defined as:

$$
D_{i}=\left(S_{o}-S_{i}\right) / S_{o} * 100 \%
$$

In the formula, $D i$ represents the importance degree of factor $i$; $S_{o}$ represents the incidence of parent nodes; $S_{i}$ represents the incidence of parent node when factor $i$ does not occur.

\section{Case Analyses}

This paper took Shipyard W in Nantong Jiangsu as the object of research. In the process of business operation, Shipyard $\mathrm{W}$ is the manufacturing could service provider. In the earlier stage, we investigated and collected all the information and data the company monitored and obtained in the process of business operation. On the basis of preliminary analysis, this paper screened and categorized influencing factors on manufacturing cloud service composition flexibility which it paid

\begin{tabular}{|c|c|c|c|c|c|}
\hline \multicolumn{2}{|c|}{ Influencing Factors Happened } & \multirow{2}{*}{$\frac{\text { Quantity }}{324}$} & \multirow{2}{*}{$\begin{array}{c}\text { Percentage } \\
(\%)\end{array}$} & \multirow{2}{*}{$\begin{array}{c}\text { Failure Rate } \\
\left(\times 10^{-4}\right) \\
0.893\end{array}$} & \multirow{3}{*}{$\begin{array}{l}\text { Note } \\
\text { COR fails } 674 \text { times, } \\
\text { accounted for } 28.39 \% \text {, } \\
\text { failure rate: } 1.857 \times \\
10^{-4}\end{array}$} \\
\hline COR & Static factors & & & & \\
\hline & Dynamic factors & 350 & 14.74 & 0.964 & \\
\hline & Competitor & 210 & 8.85 & 0.579 & COPR fails 423 times, \\
\hline \multirow[t]{4}{*}{ COPR } & Cooperator & 76 & 3.20 & 0.209 & accounted for $18.29 \%$, \\
\hline & Complementary partner & 125 & 5.27 & 0.345 & failure rate: $1.196 \mathrm{x}$ \\
\hline & Other & 23 & 0.97 & 0.063 & $10^{-4}$ \\
\hline & Network overload & 69 & 2.91 & 0.190 & BCMP fail 180 times, \\
\hline \multirow[t]{5}{*}{ BCMP } & Access authority change & 13 & 0.55 & 0.036 & accounted for $7.59 \%$, \\
\hline & System update & 28 & 1.18 & 0.077 & failure rate: $0.496 \mathrm{x}$ \\
\hline & System find threat & 70 & 2.95 & 0.193 & $10^{-4}$ \\
\hline & Timeliness & 119 & 5.01 & 0.328 & \\
\hline & Availability & 46 & 1.94 & 0.127 & PESU fails 440 times, \\
\hline \multirow[t]{4}{*}{ PSEU } & Reliability & 65 & 2.74 & 0.179 & accounted for $18.53 \%$, \\
\hline & Reputation & 20 & 0.84 & 0.055 & failure rate: $1.212 \mathrm{x}$ \\
\hline & Cost & 103 & 4.34 & 0.284 & $10^{-4}$ \\
\hline & Service & 87 & 3.66 & 0.239 & \\
\hline
\end{tabular}
special attention for. The related statistical analysis data is demonstrated in Table 1.

Table 1 the Statistical Analysis 


\begin{tabular}{llllll} 
& Task increase & 194 & 8.17 & 0.534 & CTDR fails 499 times, \\
CTDR & Task decrease & 186 & 7.83 & 0.512 & accounted for 21.02\%, \\
& Task canceled & 68 & 2.86 & 0.187 & failure rate: $1.374 \mathrm{x}$ \\
& Task restore & 51 & 2.15 & 0.141 & $10^{-4}$ \\
Other & & 147 & 6.19 & 0.405 & others \\
SUM & & 2374 & & 6.54 & \\
\hline
\end{tabular}

According to the data in Table 1 and formula (1) and (2), we can calculate each node's probability of safety (T) and failure (F), the probability value expressed as percentage, the numbers belong to the same order of magnitude with the domestic and foreign related data statistics, so it is reasonable, as shown in Fig.3:

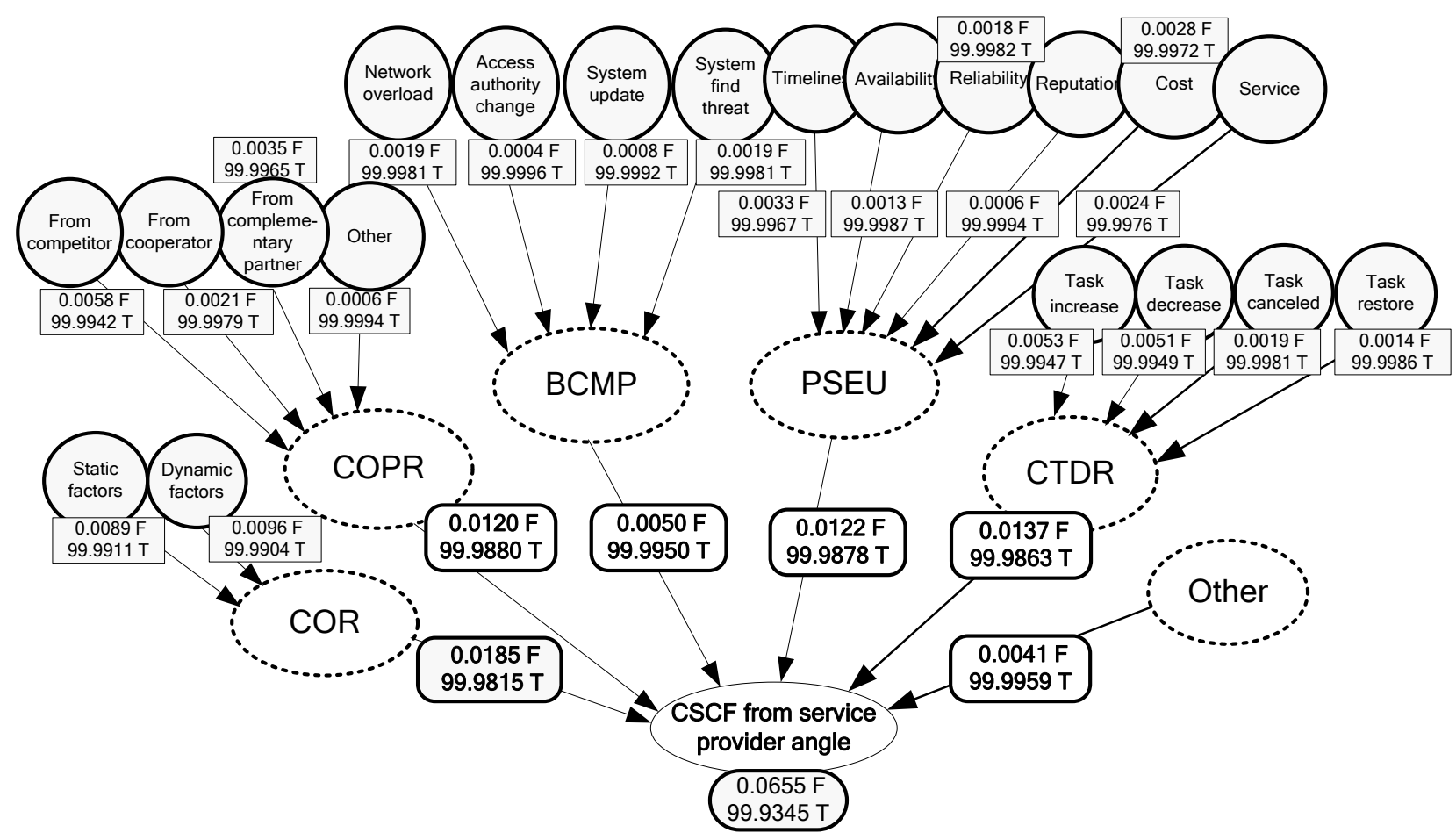

Figure. 3 Bayesian Network of CSCF from Service Provider Angle

Taking PSEU for example, we can calculate the safety probability of parent node is $99.9878 \%$, and the probability of failure is $0.0122 \%$. Similarly we can calculate the probability of other factors, and finally obtain the probability of CSCF from service provider angle failure is $6.55 \times 10^{-4}$.

In order to identify critical and uncritical influencing factors on CSCF for service provider, it is necessary to do importance analysis of each node. This paper took node "task increase" as an example. We need to reset its node status of failure (F) to 0 , assuming that tasks did not increase. The model and the rest parameters are unchanged. According to the formula (1) and (2), the failure probability of CTDR falls to $0.84 \times 10^{-4}$ from $1.37 \times 10^{-4}$, and the failure probability of CSCF from service provider angle falls to $6.02 \times 10^{-4}$ from $6.55 \times 10^{-4}$. According to formula (3), we calculated the importance degree of node "task increase" is 8.09 (The sum of all factors' importance degree is 100). Other factors' importance degree can be calculated in the same way, as shown in Fig.4: 


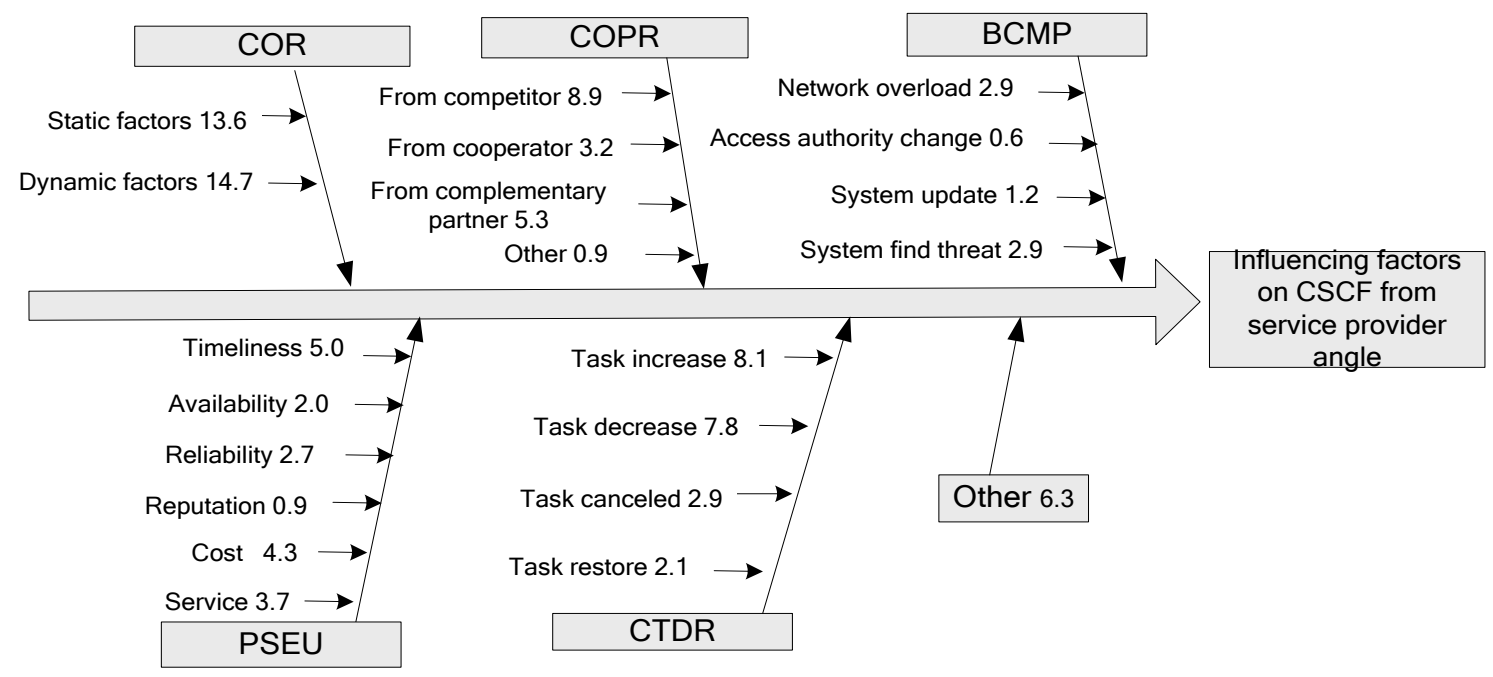

Figure. 4 Importance Degree of Influencing Factors

Fig. 4 shows us, the importance degree of note "dynamic factors" is the maximum, and the importance degree of note "access authority change" is the minimum. Through further analysis, we find that the importance degree of influence factors related to COR factors is significantly higher than other factors, and BCMP is lower than others. Therefore, the influence factors related to COR can be defined as key factors, and related to BCMP can be defined as non-critical factors.

\section{Conclusions}

In this paper, the influencing factors of CSCF were analyzed deeply from service provider angle. A Bayesian network model was built to analyze the importance degree of influencing factors on CSCF from service provider angle, and the critical and non-critical factors for service provider were identified. We find that the importance degree of influence factors related to COR factors is significantly higher than other factors, and BCMP is lower than others. Therefore, the influence factors related to COR can be defined as key factors, and related to BCMP can be defined as non-critical factors. This will put the groundwork for the subsequently monitoring of the exceptional factors and the management of the flexibility of cloud service composition. Next, we are going to research the monitoring framework and mechanism of influencing factors on cloud service composition flexibility from service provider angle.

\section{Acknowledgements}

This work was supported by the Natural Science Foundation of China (71371088) and Innovation Project of University Graduate Student in Jiangsu province (KYZZ15_0297; 1042161404).

\section{References}

[1] Bohu Li, Lin Zhang, Shilong Wang, et al. Cloud manufacturing: a new service-oriented manufacturing model [J]. Computer Integrated Manufacturing System, 2010, 16(1):1-7, 16.

[2] Lin Zhang, Yongliang Luo, Wenhui Fan, et al. Analyses of cloud manufacturing and related advanced manufacturing model [J]. CIMS 2011, 17(3):458-468.

[3] Chao Yin, Yun Zhang, Ting Zhong. Optimization model of cloud manufacturing services resource 
combination for new product development [J]. CIMS, 2012, 18(7):1368-1378.

[4] Fei Tao, Lin Zhang, Hua Guo, et al. Typical characteristics of cloud manufacturing and several key issues of cloud service composition [J]. CIMS, 2011, 17(3): 477-486.

[5] Yong Zhu, Junzhou Luo, Wei Li, et al. An approach for energy aware multipath service composition based on workflow [J]. Chinese Journal of Computers, 2012, 35(3): 627-638.

[6] Zhizhong Liu, Xiao Xue, Jiyu An, et al. Research on manufacturing cloud service optimal composition based on CL-MMAS algorithm [J]. Computer Engineering and Applications, 2012, 48(25): 239-242,248.6.

[7] Weining Liu, Bo Liu, Dihua Sun, et al. Multi-task oriented service composition in cloud manufacturing [J]. CIMS, 2013, 19(1):199-209.

[8] Le Wei, Qiuyun Zhao, Hongping Shu. Adaptive adjustment composite cloud service based on QoS for cloud manufacturing environment [J].Journal of Lanzhou University: Natural sciences, 2012, 48(4):98-104.

[9] Lianwen Zhang, Haipeng Guo, Introduction to Bayesian Network [M]. Science Press, 2006.

[10]Yusheng Hu, Xiaoyu Cui. Reasoning method of uncertainty knowledge based on Bayesian Network [J]. CIMS, 2001, 7(12): 65-68. 